\title{
A scent of therapy: Synthetic polysulfanes with improved physico-chemical properties induce apoptosis in human cancer cells
}

\author{
DANY REZK ALLAH ${ }^{1}$, LISA SCHWIND $^{1}$, IMAD ABU ASALI $^{1,3}$, JAWAD NASIM $^{2}$, \\ CLAUS JACOB $^{2}$, CLAUDIA GÖTZ ${ }^{1}$ and MATHIAS MONTENARH ${ }^{1}$ \\ ${ }^{1}$ Medical Biochemistry and Molecular Biology, Saarland University, D-66424 Homburg; \\ ${ }^{2}$ Bioorganic Chemistry, Department of Pharmacy, Saarland University, D-66123 Saarbruecken, Germany; \\ ${ }^{3}$ Laboratory Medicine Department, Faculty of Medicine, Damascus University, Damascus, Syrian Arabic Republic
}

Received May 18, 2015; Accepted June 29, 2015

DOI: 10.3892/ijo.2015.3093

\begin{abstract}
Diallyl sulfanes derived from edible plants are highly potent compounds which at sub-millimolar concentrations are able to induce the formation of reactive oxygen species (ROS) in a variety of different cells, where they often cause an altered redox status. The loss of cellular thiols and/or formation of ROS subsequently triggers a range of cellular responses, including the induction of apoptosis. A great disadvantage of natural diallyl mono- and polysulfanes, however, is their inherent insolubility in water and the extremely bad odour which limits their practical use in humans. Here, we present the synthesis and biological evaluation of two new, especially designed polysulfanes, namely the trisulfide 1-Allyl-3-(2-ethoxyethyl)trisulfide (ATSEE) and the tetrasulfide Allyl-4-benzyltetrasulfide (ATTSB), which are nearly odourless. Both compounds produce $\mathrm{O}_{2}{ }^{--}$radicals in HCT116 cells and both induce an oxidative defence signalling. Cell viability is especially reduced by the tetrasulfane ATTSB, with an arrest of the cell cycle in the $\mathrm{G}_{2}$-phase. In contrast, the trisulfane ATSEE does not inhibit the cell cycle. In agreement with these findings, treatment of HCT116 cells with ATTSB ultimately results in apoptosis whereas only limited induction of apoptosis has been detected for cells treated with ATSEE. We further show that antioxidative defence mechanisms and death response signalling run in parallel and the dominant pathway decides the fate of the cell. Thus, our results not only illuminate the intricate mode of action of certain polysulfanes; they also demonstrate that the new odourless tri- and tetrasulfanes exhibit a similar activity compared to their natural
\end{abstract}

Correspondence to: Professor Mathias Montenarh, Medical Biochemistry and Molecular Biology, Saarland University, Building 44, D-66424 Homburg, Germany

E-mail: mathias.montenarh@uks.eu

Key words: polysulfanes, reactive oxygen species, thiols, apoptosis oxydative defence, cell cycle, cell viability counterparts, yet are easier to handle and also deprived of the offensive odour which so far has prevented most practical applications of such polysulfanes, at least in the context of medicine.

\section{Introduction}

A wide range of epidemiologic studies conducted during the last couple of decades has shown that the dietary intake of Allium vegetables, such as garlic and onions, may reduce the risk of various types of malignancies including cancer. Among the different secondary metabolites present in Allium vegetables, organosulfur compounds stand out and frequently are considered as the agents responsible for the diverse beneficial activities associated with these plants (1). These compounds have been known since the 19th century, when chemists distilled several sulfur containing compounds from garlic, notably diallyl mono- and polysulfanes $(2,3)$. Such compounds are chemically comparably 'simple' and procedures are now available to synthesize diallyl trisulfane (DATS) and diallyltetrasulfane (DATTS), employing the methods of Milligan et al (4) and Derbesy and Harpp (5), respectively. The chemical synthesis provides these compounds in high quantities and with great purity. Indeed, our own group has used and recently refined these traditional synthetic procedures in order to synthesize a range of new symmetric and asymmetric polysulfanes, often with especially designed properties $(6,7)$. The availability of such pure compounds in good yields has also stimulated the analysis of various biological activities of individual compounds, especially, but not exclusively, in the context of cancer research $(8,9)$. As part of these studies, the importance of the sulfur-sulfur chain length and choice of side chains has been established, and some aspects of the underlying cellular mode(s) of action and potential cellular targets have been revealed. Over the last 20 years it has been shown, for instance, that polysulfanes are able to modulate the intracellular redox system either by the production of reactive oxygen species (ROS) or by oxidizing thiol groups of cellular proteins, leading to the formation of mixed disulfides (reviewed in refs. 10,11). It is still unclear if the observed 
increase in ROS is actually a cause or rather a consequence of a diminished cellular thiol content - or vice versa. Yet it appears that the cellular thiolstat is the prime target of such compounds, a finding which incidentally is confirmed by the present study, and that a modulation of this thiolstat goes hand in hand with diverse cellular stresses, stress response and signalling mechanisms.

Indeed, mono- and polysulfanes modulate a number of different intracellular signalling pathways including those regulating the cell cycle, ER-stress response and apoptosis (reviewed in refs. 11,12). Interestingly, it has also been shown by a number of independent studies that cancer cells are much more sensitive to the treatment with diallyl mono- and polysulfides when compared to normal cells. Here, cancer cells usually die by apoptosis whereas normal healthy cells survive (13-16). Whilst such selectivity for cancer cells is highly unusual for molecules derived from non-toxic, even edible plants, it can be explained in part by the particular predisposition of cancer cells to react highly sensitively to externally induced changes in their intracellular redox state $(10,17)$. Not surprisingly, compounds such as DATS and DATTS have long been considered as potential anticancer drugs, yet practical applications have been hampered by their low solubility in aqueous media, a certain chemical instability at room temperature, and, above all, their purgent smell.

A couple of years ago, we therefore embarked on the synthesis of polysulfane derivatives which retain the original biological activity associated with DATS and DATTS, yet also exhibit properties which allow a wider application of such polysulfanes, especially in the context of medicine.

Here, we report the synthesis and biological evaluation of two new polysulfanes which have been designed specifically for practical applications in the field of medicine. These compounds are easy to synthesize and to handle, and whilst they are devoid of any offensive odour, they still retain the ability to attack HCT116 cancer cells via an intricate mechanism of intracellular redox modulation.

\section{Materials and methods}

Reagents andantibodies.Protease inhibitor cocktail complete ${ }^{\mathrm{TM}}$ was purchased from Roche Diagnostics, Mannheim, Germany. Dimethyl sulfoxide (DMSO) was obtained from Merck, Darmstadt, Germany. Antibodies against GAPDH and Nrf2 were from Santa Cruz Biotechnology (Heidelberg, Germany). Anti-poly(ADP-ribose) polymerase (anti-PARP) antibodies and anti-heme oxygenase-1 (anti-HO-1) antibodies were purchased from Cell Signaling Technology (Frankfurt, Germany). Ellman's reagent was purchased from Thermo Scientific (Schwerte, Germany). Goat, mouse and rabbit secondary antibodies were all from Dianova (Hamburg, Germany). Unless otherwise stated, the reagents for the chemical synthesis were from Sigma-Aldrich (Munich, Germany) and used without further purification.

Chemical analysis of synthetic compounds. NMR spectra were recorded on a Bruker Avenue 500. The measuring frequency was $500 \mathrm{MHz}$ for ${ }^{1} \mathrm{H} \mathrm{NMR}$ and $125 \mathrm{MHz}$ for ${ }^{13} \mathrm{C} \mathrm{NMR}$. Measurements were performed at room temperature and $\mathrm{CDCl}_{3}$ and DMSO were used as solvent. Liquid chromatography coupled with mass spectroscopy (LCMS) was performed on a Bischoff Lambda $1000 \mathrm{UV} /$ Vis at $275 \mathrm{~nm}$ using a YMCC 18 column and methanol/water (85:15) as mobile phase at a flow rate of $1.0 \mathrm{ml} / \mathrm{min}$. Analytical HPLC analysis was performed using an Agilient HP1050 series HPLC system in combination with a Zorbax $\mathrm{C} 18$ column $(150 \times 4.6 \times 5 \mathrm{~mm})$ and a security guard $\mathrm{C} 18$ pre-column and with a diode array detection. The auto-sampler temperature was $4^{\circ} \mathrm{C}$ and the column temperature was $37^{\circ} \mathrm{C}$. The cut-off pressure was 280 bar. Detection was performed at $254 \mathrm{~nm}$. The optimal injection volume of each sample was $10 \mu \mathrm{l}$. Prior to analysis, compounds were dissolved in HPLC grade solvents.

Synthesis of polysulfanes. DATS and DATTS were synthesized following the methods described by Milligan et al and by Derbesy and Harpp, respectively $(4,5)$. These literature methods have been modified by us to enable the synthesis of asymmetric tri- and tetrasulfanes $(6,7)$.

1-Allyl-3-(2-ethoxyethyl)trisulfane (ATSEE) was synthesized according to a modified method relying on the original method of Milligan et al (4). Solvent $(20 \mathrm{ml})(60 \% \mathrm{v} / \mathrm{v}$ ethanolwater) was added to a clean round bottom flask. Sodium thiosulfate pentahydrate $(6.2 \mathrm{~g}, 25 \mathrm{mmol}), 2$-chloroethylethyl ether $(1.09 \mathrm{~g}, 10 \mathrm{mmol})$ and allyl chloride $(0.765 \mathrm{~g}, 10 \mathrm{mmol})$ were added to the flask. The mixture was subjected to reflux until it became homogeneous. Then ethanol was removed by distillation under reduced pressure and the impurities were extracted using petroleum ether. Afterwards, the remaining aqueous phase was treated with an aqueous solution of sodium sulfide $(0.78 \mathrm{~g}, 10 \mathrm{mmol}$ in $20 \mathrm{ml}$ distilled water). The mixture was stirred for $45 \mathrm{~min}$ at room temperature. The product was extracted using petroleum ether. The organic layer of petroleum ether was dried over anhydrous sodium sulfate, filtered and the solvent was evaporated under reduced pressure. The compound was purified by column chromatography using petroleum ether / ethyl acetate (95:5) as eluent $\left(\mathrm{TLC} \mathrm{R}_{f}=0.22\right)$. ATSEE was obtained as a yellow viscous oil with $10 \%$ overall yield $(0.21 \mathrm{~g}, 0.1 \mathrm{mmol})$.

${ }^{1} \mathrm{H}$ NMR $\left(500 \mathrm{MHz}, \mathrm{CDCl}_{3}\right): \delta \mathrm{ppm}=5.92-5.79(\mathrm{~m}, 1 \mathrm{H}$, $\mathrm{CH})$, 5.25-5.11 (m, 2H, 1- $\left.\mathrm{CH}_{2}\right), 3.76-3.62\left(\mathrm{~m}, 2 \mathrm{H}, 1-\mathrm{CH}_{2}\right)$, 3.55-3.47 (m, 4H, 2- $\left.\mathrm{CH}_{2}\right), 3.07-3.02$ (m, 2H, 1- $\left.\mathrm{CH}_{2}\right), 1.24-1.16$ $\left(\mathrm{m}, 3 \mathrm{H}, 1-\mathrm{CH}_{3}\right) ;{ }^{13} \mathrm{C} \mathrm{NMR}\left(125 \mathrm{MHz}, \mathrm{CDCl}_{3}\right): \delta \mathrm{ppm}=132.66$ (1-C), 119.13 (1-C), 68.47 (1-C), 66.56 (1-C), 41.42 (1-C), 38.37 (1-C), 15.14, (1-C); LCMS: 25 min retention time; MS (EIMS) $\mathrm{m} / \mathrm{z}$ found 210.01 , calculated 210.02 .

1-Allyl-4-benzyltetrasulfane (ATTSB) was synthesized according to a modified method based on the original procedure reported by Derbesy and Harpp (5). A solution of allyl mercaptan $(0.56 \mathrm{~g}, 15 \mathrm{mmol})$ and pyridine $(1.2 \mathrm{ml}, 15 \mathrm{mmol})$ in diethyl ether $(37.5 \mathrm{ml})$ was placed into a dropping funnel and added slowly over a period of $30 \mathrm{~min}$ to a stirred solution of disulfur dichloride $\mathrm{S}_{2} \mathrm{Cl}_{2}(1.2 \mathrm{ml}, 15 \mathrm{mmol})$ in diethyl ether $(75 \mathrm{ml})$, which was kept at $-78^{\circ} \mathrm{C}$ under argon. The solution was stirred at $-78^{\circ} \mathrm{C}$ for $45 \mathrm{~min}$, at which point a solution of benzyl mercaptan $(0.93 \mathrm{~g}, 15 \mathrm{mmol})$ and pyridine $(1.2 \mathrm{ml}, 15 \mathrm{mmol})$ in diethyl ether $(37.5 \mathrm{ml})$ was added dropwise over a period of $30 \mathrm{~min}$. The reaction mixture was stirred for further $1 \mathrm{~h}$ and subsequently allowed to warm up slowly to room temperature. The mixture was then washed with distilled water $(2 \times 25 \mathrm{ml})$, $1 \mathrm{M}$ aqueous $\mathrm{NaOH}$ solution $(2 \times 25 \mathrm{ml})$ and again with distilled 
water $(2 \times 25 \mathrm{ml})$ to remove impurities. The $\mathrm{pH}$ was adjusted to neutral with $1 \mathrm{M}$ aqueous $\mathrm{NaOH}$ solution. The aqueous phase was separated and discarded, whilst the organic phase containing the product was dried over anhydrous $\mathrm{Na}_{2} \mathrm{SO}_{4}$, filtered and the solvent evaporated under reduced pressure. The target compound was purified further by column chromatography using petroleum benzene: ethyl acetate (95:5) as eluent (TLC; $\mathrm{R}_{f}$ 0.57). The title compound was obtained as a yellow oil in $17 \%$ overall yield $(0.5 \mathrm{~g}, 2.04 \mathrm{mmol})$.

${ }^{1} \mathrm{H}$ NMR $(500 \mathrm{MHz}, \mathrm{DMSO}): \delta \mathrm{ppm}=7.36-7.25(\mathrm{~m}$, 5H, Ar), 5.91-5.80 (m, 1H, 1-CH), 5.28-5.17 (m, 2H, 1- $\left.\mathrm{CH}_{2}\right)$, 4.21-4.14 (s, 2H, $\left.1 \mathrm{CH}_{2}\right), 3.62-3.53\left(\mathrm{~m}, 2 \mathrm{H}, 1-\mathrm{CH}_{2}\right) ;{ }^{13} \mathrm{C} \mathrm{NMR}$ (125 Hz, DMSO): $\delta$ ppm = 132.47 (1-C), 129.55 (2-C), 128.70 (2-C), 127.70 (1-C), 119.94 (1-C), 119.55 (1-C), 43.53 (1-C), 42.05 (1-C); LCMS: 10 min retention time; MS (EIMS) m/z found 259.0 , calculated 259.88 .

Cell culture. HCT116 cells (ATCC number: CCL-247) were maintained at $37^{\circ} \mathrm{C}$ and $5 \% \mathrm{CO}_{2}$ in McCoy's 5A medium (PromoCell, Heidelberg, Germany) with $10 \%$ fetal calf serum (FCS). The polysulfanes were dissolved in DMSO to $50 \mathrm{mM}$ stock solutions, which were prepared freshly before use.

Determination of cell viability. HCT116 cells were seeded at a concentration of $1 \times 10^{4}$ cells per well to a final volume of $500 \mu \mathrm{l}$ in a 24-well plate and incubated overnight at $37^{\circ} \mathrm{C}$ and $5 \% \mathrm{CO}_{2}$ in a humidified atmosphere. Cells were incubated with $50 \mu \mathrm{M}$ ATSEE or ATTSB for 24, 48 and 72 h. Cell viability was determined by the colourimetric MTT (3-(4,5)-dimethylthiazol-2-yl)-2,5 diphenyltetrazolium bromide (Sigma, Germany) assay according to the manufacturer's instructions. One hour before the end of treatment $50 \mu \mathrm{l}$ MTT $(5 \mathrm{mg} / \mathrm{ml}$ PBS) were added. Following 1-h treatment with MTT, the medium was disposed and cells were solubilized by adding $500 \mu \mathrm{l}$ solubilizing solution $[10 \%(\mathrm{w} / \mathrm{v})$ SDS in DMSO and $0.6 \%(\mathrm{v} / \mathrm{v})$ acetic acid] to each well. The absorbance of the purple-blue formazan dye was determined in an ELISA reader at $595 \mathrm{~nm}$.

Cell cycle analysis. HCT116 cells were seeded in a concentration of $5 \times 10^{5}$ cells and grown in a $6-\mathrm{cm}$ petri dish overnight. Cells were treated with $50 \mu \mathrm{M}$ ATSEE or $50 \mu \mathrm{M}$ ATTSB for 48 h. Cells were collected and washed two times with cold PBS and $5 \mathrm{mM}$ EDTA before being resuspended in PBS and EDTA and fixed with $70 \%$ ethanol. Cells were treated with RNAse and propidium iodide to label DNA. Cells were analysed by flow cytometry immediately after staining.

Annexin $V$ staining. Annexin $\mathrm{V}$ staining was performed with the Alexa Fluor ${ }^{\circledR} 488$ Annexin V/Dead cell apoptosis kit from Life Technologies (Darmstadt, Germany). Briefly, HCT116 cells were grown on polylysine coated cover slips for $24 \mathrm{~h}$. Then cells were either treated with $50 \mu \mathrm{M}$ ATTSB, $50 \mu \mathrm{M}$ ATSEE or the solvent control DMSO. After incubation for $24 \mathrm{~h}$, medium was removed and cells were washed three times with cold PBS and then incubated with Alexa Fluor 488 labelled Annexin V for $15 \mathrm{~min}$. Cells were washed and finally analysed under a fluoroscence microscope.

Extraction of cells. Following incubation of HCT116 cells with either ATSEE or ATTSB, cells were collected in cold phos- phate-buffered saline (PBS) and centrifuged at $4^{\circ} \mathrm{C}$ and $250 \mathrm{x} \mathrm{g}$ for $5 \mathrm{~min}$. After another washing step with cold PBS, cells were lysed with 50-150 $\mu$ l of RIPA buffer [50 mM Tris-HCl, pH 8.0, $150 \mathrm{mM} \mathrm{NaCl}, 0.5 \%$ sodium desoxycholate, $1 \%$ Triton $\mathrm{X}-100$, $0.1 \%$ sodium dodecylsulfate (SDS)] supplemented with the protease inhibitor cocktail complete. The cell lysate was left on ice for $15 \mathrm{~min}$ and then subjected to sonification (3x1 min) at $4^{\circ} \mathrm{C}$. Cell debris was removed by centrifugation at $16000 \mathrm{x} \mathrm{g}$ at $4^{\circ} \mathrm{C}$ for $30 \mathrm{~min}$. The protein content of the supernatant was determined by the Bradford method using the Bio-Rad protein assay reagent (Bio-Rad, Munich, Germany).

For the analysis of the nuclear fraction of Nrf2, cells were washed with cold PBS and then thoroughly resuspended in 50-150 $\mu$ l cytosolic lysis buffer (10 mM HEPES-KOH, $\mathrm{pH} 7.9$, $1.5 \mathrm{mM} \mathrm{MgCl}_{2}, 10 \mathrm{mM} \mathrm{KCl}, 0.5 \mathrm{mM}$ DTT and $0.5 \% \mathrm{NP} 40$ ) supplemented with freshly prepared protease inhibitor cocktail complete. The cell lysate was left on ice for $20 \mathrm{~min}$ before being centrifuged at $16000 \mathrm{x}$ g at $4^{\circ} \mathrm{C}$ for $5 \mathrm{~min}$. The supernatant (cytosolic fraction) was removed and the pellet lysed with 50-150 $\mu$ l nuclear lysis buffer (10 mM HEPES-KOH, pH 7.9, $25 \%$ glycerol, $420 \mathrm{mM} \mathrm{NaCl}, 1.5 \mathrm{mM} \mathrm{MgCl}_{2}, 0.2 \mathrm{mM}$ EDTA and freshly prepared $0.5 \mathrm{mM}$ DTT and $0.5 \%$ Triton X-100) supplemented with protease inhibitor complete. After removal of the cell debris the supernatant was analysed further by SDS polyacrylamide gel electrophoresis followed by western blotting.

SDS-polyacrylamide gel electrophoresis and western blot analysis. Proteins of total cell extract (50-200 $\mu \mathrm{g}$ ) were separated by size using either a 10 or $12.5 \%$ SDS polyacrylamide gel. Following electrophoresis, proteins were transfered to polyvinylidene difluoride (PVDF) membranes by tank blotting with $20 \mathrm{mM}$ Tris- $\mathrm{HCl}, \mathrm{pH} 8.8,150 \mathrm{mM}$ glycine as transfer buffer. To ensure equal loading of samples, GAPDH, nucleolin or $\alpha$-tubulin were used as loading controls. Subsequent immunoblotting was performed by blocking non-specific binding regions of the membrane with 5\% skimmed milk in PBS with $0.1 \%$ Tween-20 for $1 \mathrm{~h}$ at room temperature. The goat polyclonal anti-Nrf2, rabbit polyclonal anti-HO-1, anti-PARP, anti-nucleolin, and the mouse monoclonal anti- $\alpha$-tubulin antibodies were each separately used in a dilution of 1:1,000 overnight with gentle shaking at $4^{\circ} \mathrm{C}$. The rabbit polyclonal GAPDH antibody was used in a dilution of 1:1,000 at room temperature for $1 \mathrm{~h}$. After washing the membranes with PBS-Tween-20 containing $1 \%$ skimmed milk ( $3 \times 10 \mathrm{~min})$, they were then incubated with the respective peroxidase-coupled secondary antibodies in appropriate dilutions at room temperature. The membranes were washed further (3x10 min) in PBS-Tween-20. Signals were developed and visualised by the Lumilight system of Roche Diagnostic (Mannheim, Germany).

Determination of $\mathrm{O}_{2}{ }^{-*}$ in cell culture. Production of the superoxide radical was analysed by oxidation of dihydroethidium (DHE) to 2-hydroxyethidium. Cells were seeded in 96-well plates and grown overnight. After removal of the cell culture medium, cells were washed with PBS, and $190 \mu \mathrm{l}$ of $25 \mu \mathrm{M}$ DHE dissolved in PBS was added to each well. Immediately before the measurement was started, increasing concentrations of the polysulfane or DMSO ( $0.5 \%$ final concentration) as solvent control were added. The dosage-dependent increase in 


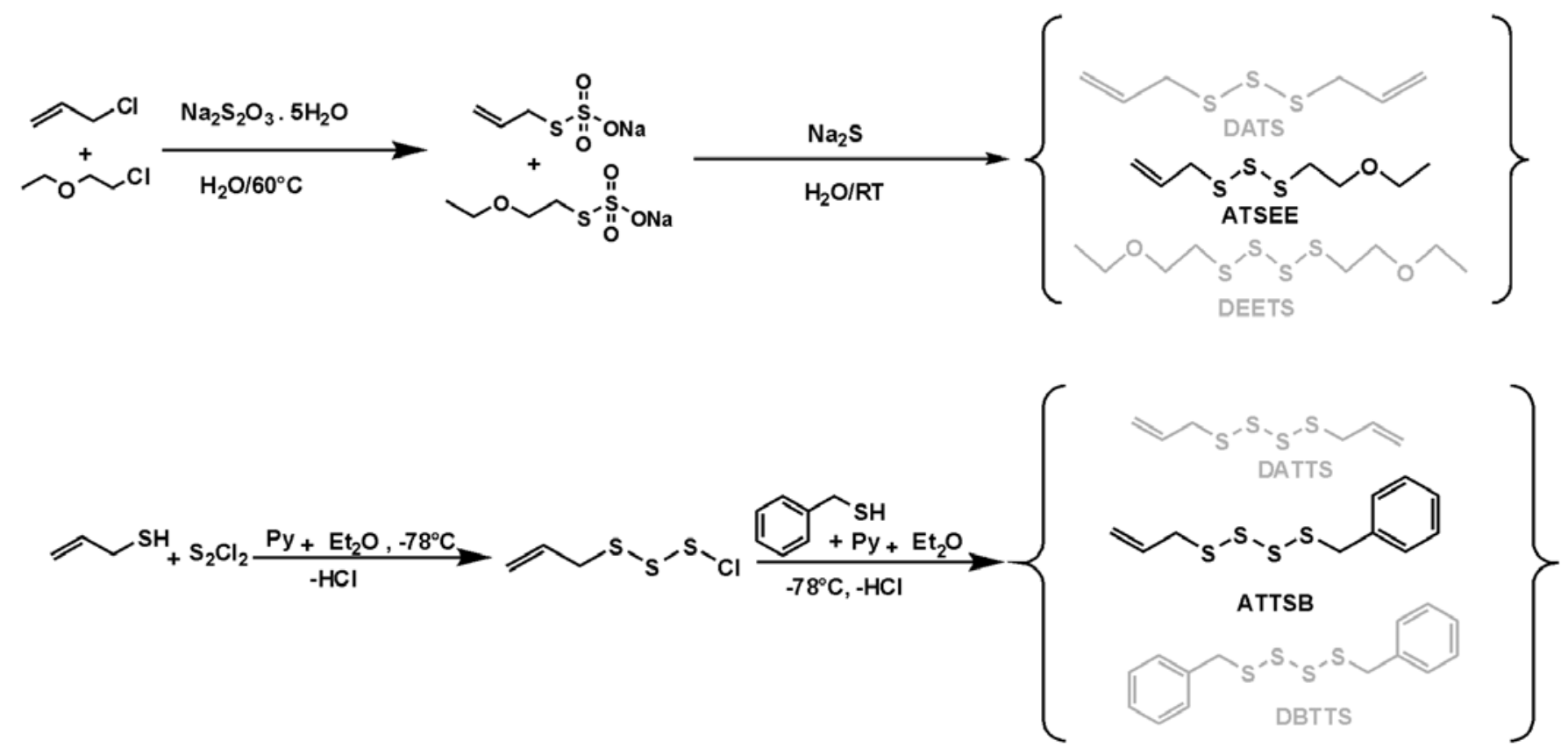

Figure 1. Synthesis of ATSEE and ATTSB, the asymmetric trisulfane and tetrasulfane used in this study. The methods employed necessarily also result in the formation of the symmetric by-products, namely DATS and diethoxyethyltrisulfane (DEETS) in the case of ATSEE and DATTS and dibenzyltetrasulfane (DBTTS) in the case of ATTSB. Further details are provided in the text.

fluorescence (DHE ex/em: 563/587 nm) was measured using a Tecan infinite M200 device.

Determination of total thiol content. Total intracellular thiol levels were measured as previously described (15). Briefly, HCT116 cells were seeded in $10-\mathrm{cm}$ cell culture dishes $\left(1 \times 10^{6}\right.$ cells/dish) and grown overnight. Medium was then changed and cells were treated with $50 \mu \mathrm{M}$ of polysulfanes for $0,5,10,20,30,60,120$ or $240 \mathrm{~min}$. After removal of the cell culture medium, cells were washed with PBS, collected in $250 \mu \mathrm{l} \mathrm{PBS}$, and snap-frozen in liquid nitrogen. Following thawing and removal of cellular fragments by centrifugation at $12,000 \mathrm{x} \mathrm{g}$ for $10 \mathrm{~min}$ at $4^{\circ} \mathrm{C}$, the total thiol content of cell lysates was determined using 5,5'-dithiobis(nitrobenzoic acid) (Ellman's reagent) and cysteine hydrochloride monohydrate as a standard.

Statistical analysis. Results are represented as means \pm SD. For statistical evaluation, Student's t-test was applied using Origin 6.1 with $\mathrm{p}<0.05$ considered as significant, marked with one asterisk or $\mathrm{p}<0.01$ considered as very significant, marked with two asterisks in the figures.

\section{Results}

In the first step, several tri- and tetrasulfanes were especially designed, synthesized and pre-screened for cytotoxicity. Among these compounds, two new polysulfanes, namely the trisulfide 1-Allyl-3-(2-ethoxyethyl)trisulfide (ATSEE) and the tetrasulfide Allyl-4-benzyltetrasulfide (ATTSB), were selected for further studies due to their pronounced activity. Both compounds are asymmetric (see Fig. 1 for chemical structures). These molecules still feature the allyl group which may be important for biological activity yet they also contain another function which is deemed essential for solubility and reduced volatility, and hence odour.

As part of the synthetic procedures, the classical procedure of Derbesy and Harpp (5) and of Milligan et al (4), respectively, have been modified in order to produce the relevant asymmetric polysulfanes $(6,7)$. The analytical data obtained for both compounds is in agreement with their structure and indicate a purity of $\geq 94 \%$ for ATSEE and $\geq 95 \%$ for ATTSB (see Materials and methods). The yields obtained so far are low, but this is due to the fact that the synthetic method has not yet been optimized and that the formation of symmetric 'by-products' unfortunately is unavoidable. As anticipated, both compounds are nearly odourless. ATSEE is soluble in water and in DMSO, yet the more lipophilic compound ATTSB is soluble only in DMSO. Whilst future studies may well consider improving the synthetic yield of these compounds, the crude method employed here was adequate to obtain sufficient product for subsequent activity screens and cell biological investigations.

In the next step, we addressed the question whether ATSEE and ATTSB might have an effect on cancer cells. The activity of both compounds was evaluated in cultured HCT116 human colon cancer cells and compared directly to the corresponding activity of the well-known natural analogues DATS and DATTS. As part of this study, HCT116 cells were treated with $50 \mu \mathrm{M}$ of ATSEE and ATTSB as well as DATS and DATTS for 24, 48 and $72 \mathrm{~h}$. Cell viability was determined with an MTT assay and DMSO was used as solvent control. As shown in Fig. 2 for all four compounds used, cell viability was reduced to $\sim 40 \%$ after $24 \mathrm{~h}$ compared to the solvent control. There was a further reduction in cell viability 48 and $72 \mathrm{~h}$ after treatment. Whilst reduction of cell viability was nearly in the same range for all four compounds, there were minor differences between the tri- and tetrasulfanes. The new tetrasulfane ATTSB repeat- 


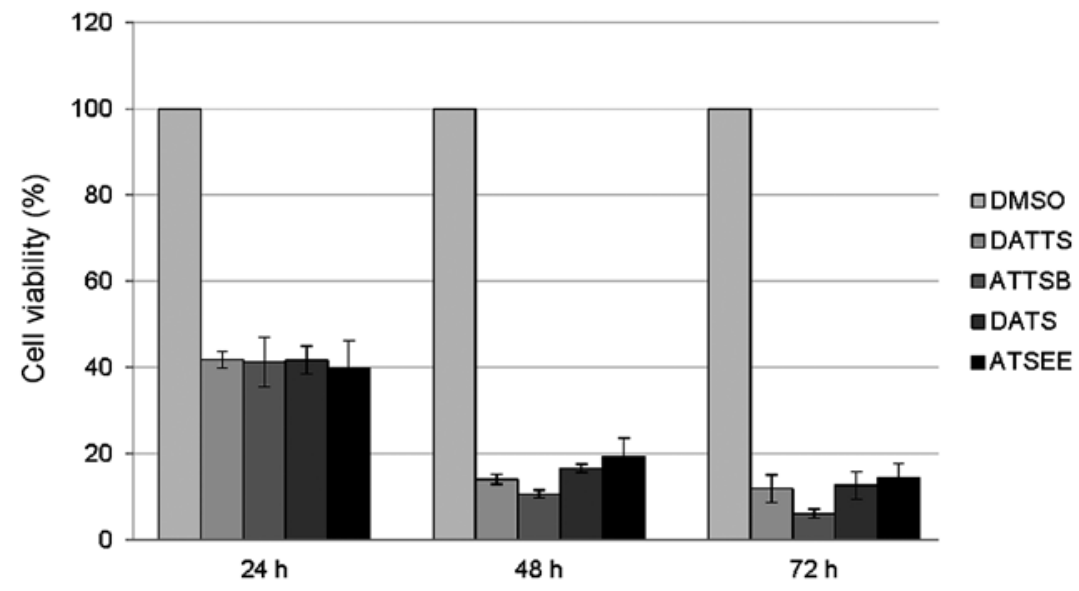

Figure 2. Comparison of viability of HTC116 cells treated with ATTSB, ATSEE, DATTS and DATS. Cells were seeded in 24-well plates and treated with $50 \mu \mathrm{M}$ ATTSB, ATSEE, DATTS, DATS or the solvent control DMSO for 24,48 or $72 \mathrm{~h}$. They were then analysed for viability by an MTT assay. The DMSO control was set to $100 \%$ and all other data calculated in relation to the DMSO control. Data represent the average of three independent experiments.

edly turned out to be the most active compound, in agreement with previous reports which consistently showed an increased activity of tetra- over trisulfanes. ATTSB was therefore identified as a possible lead compound for wider activity screens, for instance in the context of auto-inflammatory diseases, and also for future synthetic efforts aiming at the next generation of polysulfane derivatives.

Subsequently, we asked why ATSEE and ATTSB are active, and whether both might have an influence on the cell cycle of HCT116 cells. In order to address this question, HCT116 cells were incubated with either $50 \mu \mathrm{M}$ ATTSB, $50 \mu \mathrm{M}$ ATSEE or, as a control, with DMSO for $48 \mathrm{~h}$. They were then analysed by flow cytometry. As shown in Fig. 3, incubation of HCT116 cells with $50 \mu \mathrm{M}$ ATTSB for $48 \mathrm{~h}$ led to a $\mathrm{G}_{2}$ arrest. In contrast, treatment of the cells with $50 \mu \mathrm{M}$ ATSEE for $48 \mathrm{~h}$ had no effect on the cell cycle distribution, yet there was an overall reduction of cells in all cell cycle phases with an increase in the sub- $\mathrm{G}_{1}$ fraction. Thus, we conclude that the tetrasulfane ATTSB acts on cell cycle progression with a pronounced $\mathrm{G}_{2}$ arrest.

A reduction in cell number can be due to the induction of apoptosis, a process studied extensively for many natural organic sulfur compounds $(6,13,16,18)$. To investigate this possibility, we treated HCT116 cells with $50 \mu \mathrm{M}$ ATTSB or $50 \mu \mathrm{M}$ ATSEE. For comparison, we also incubated cells with the same concentration of DATTS or DATS (DMSO served as solvent control). Cells were harvested and proteins were extracted after 24 or after $48 \mathrm{~h}$. Proteins were separated in an SDS-polyacrylamide gel and PARP was detected by western blotting. As shown in Fig. 4A, after $24 \mathrm{~h}$ of treatment we already found a faint band for the PARP cleavage product $(89 \mathrm{kDa})$ which was notably absent in the DMSO control. After $48 \mathrm{~h}$ we detected a strong band for the PARP cleavage product in cells treated with ATTSB, DATTS and DATS. In contrast, only a very faint protein band for the PARP cleavage product was visible for ATSEE treated cells. Thus, it appears that ATTSB exhibits an activity which is comparable to its natural analogue, DATTS, whereas ATSEE seems to be less active in inducing apoptosis than the corresponding trisulfane DATS.
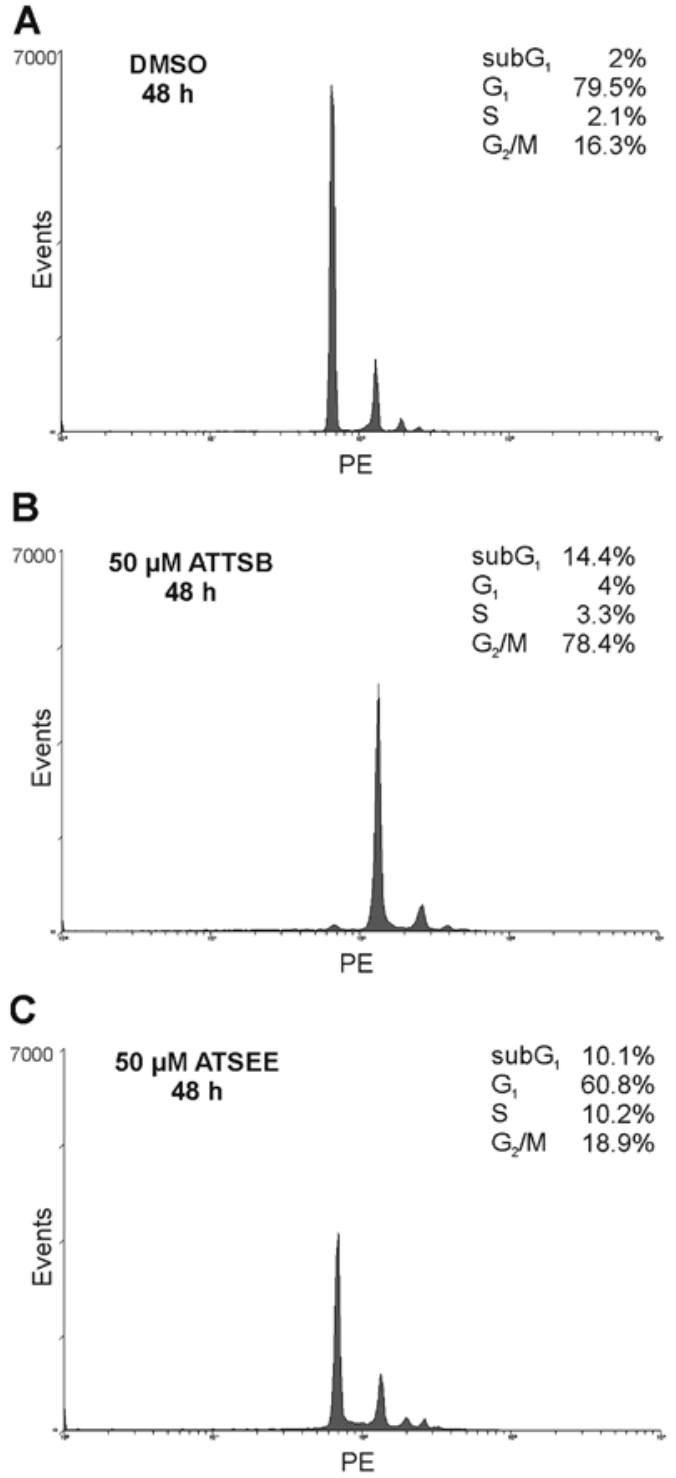

Figure 3. Influence of ATTSB and ATSEE on cell cycle distribution. HCT116 cells were treated for $48 \mathrm{~h}$ with DMSO (solvent control), $50 \mu \mathrm{M}$ ATTSB or $50 \mu \mathrm{M}$ ATSEE and then analysed by cytofluorimetry using propidium iodide (PE) to stain the DNA. One representative of three independent experiments is shown. 
A
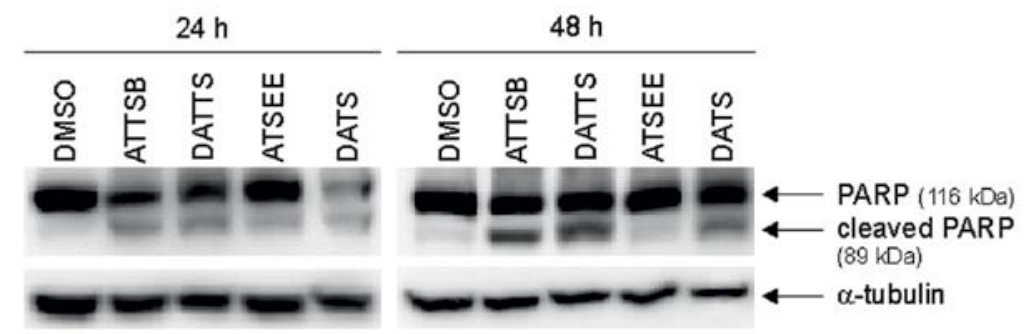

B
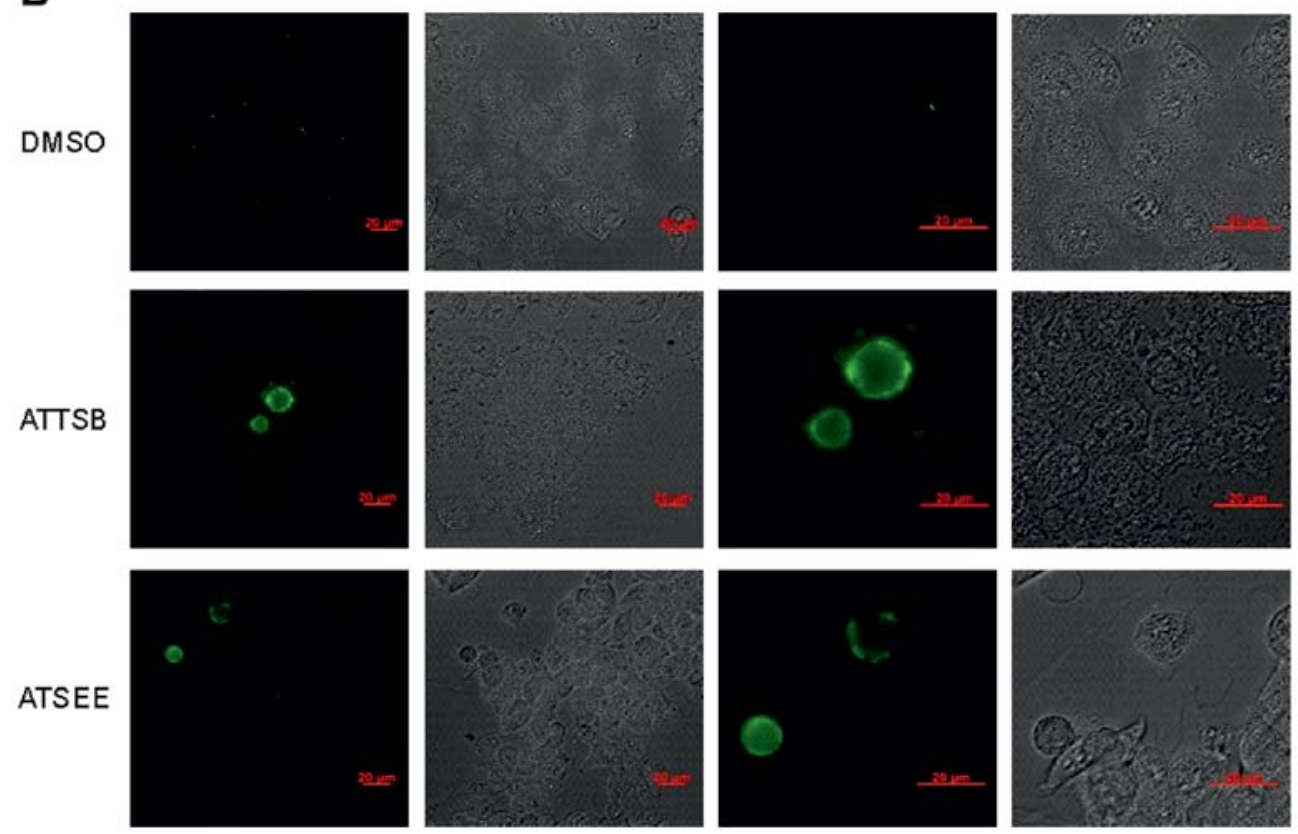

Figure 4. (A) Analysis of PARP cleavage in HCT116 cells treated with ATTSB, ATSEE, DATTS and DATS for 24 or 48 h. HCT116 cells were treated with the DMSO solvent control or either $50 \mu \mathrm{M}$ ATTSB, ATSEE, DATTS or DATS for 24 or for $48 \mathrm{~h}$. Proteins from cell extracts were analysed on a $7.5 \%$ SDS polyacrylamide gel and blotted onto a PVDF membrane. PARP and its cleavage product were identified with a PARP specific antibody (1:1,000). A representative blot of at least three western blots is presented. $\alpha$-tubulin is shown as a loading control. (B) Annexin V staining of HCT116 cells treated with ATTSB or ATSEE dissolved in DMSO. HCT116 were grown on cover slips and incubated with $50 \mu \mathrm{M}$ ATSEE or ATTSB for $24 \mathrm{~h}$. Cells were analysed for Annexin V staining by a Zeiss fluorescence microscope (cell observer). Magnification was either x 400 or x1,000.

Whilst these studies already provided a strong indication that ATTSB induces cell cycle arrest and apoptosis in HCT116 cells, we decided to confirm the induction of apoptosis by another, independent method. Therefore, Annexin V staining and analysis via fluorescence microscopy was performed. Here, HCT116 cells were treated with ATSEE or with ATTSB for $24 \mathrm{~h}$ as described above. Cells were then incubated with Alexa Fluor $488 \mathrm{~nm}$ labelled Annexin $\mathrm{V}$ and analysed by fluorescence microscopy. As shown in Fig. 4B, cells treated with ATSEE or ATTSB for $24 \mathrm{~h}$ showed positive Annexin V staining. This supports our previous findings that both compounds induce apoptosis, although with different efficiency.

Recent studies have indicated that diallyl polysulfanes are able to cause oxidative stress in specific cell lines, and that such a build-up of reactive species may be one of the events eventually leading to apoptosis. Therefore, we considered whether the new asymmetric compounds might also stimulate an increase in intracellular ROS concentrations. HCT116 cells were treated with ATSEE or with ATTSB for 10,30 and $60 \mathrm{~min}$ and analysed for increased intracellular $\mathrm{O}_{2}{ }^{-}$concentrations with the dihydroethidium (DHE) assay according to the manufacturer's instructions. We observed a time-dependent increase in the level of $\mathrm{O}_{2}{ }^{--}$for both compounds (Fig. 5A and $\mathrm{B})$. In cells treated with DMSO alone we did not detect an increase in the level of $\mathrm{O}_{2}^{-}$(Fig. 5C).

It is known for quite some time, that increases in intracellular ROS levels often go hand in hand with alterations in the intracellular thiol level, and that such an attack on the "cellular thiolstat' ultimately may be responsible for the induction of apoptosis $(19,20)$. Therefore, we investigated whether treatment of HCT116 cells with ATSEE or ATTSB might alter the total thiol concentration in these cells, bearing in mind that the latter is only a very crude reflection of individual thiol-based redox systems inside the cell and its various compartments (21). In order to estimate the 'global' thiol status of the HCT116 in response to the polysulfanes, HCT116 cells were treated with ATSEE or ATTSB for 10, 20, 30, 60 and 120 min and then the thiol concentration was measured using Ellman's reagent [5,5'-dithiobis (nitrobenzoic acid)] (22). As may be expected and shown in Fig. 6, cells treated with ATTSB showed a reduction of the total thiol concentration, whilst a smaller decrease could be observed in cells treated with ATSEE. Interestingly, the reduction in thiol levels was of the same range compared to the increase in ROS levels. It is also notable that the reduction 

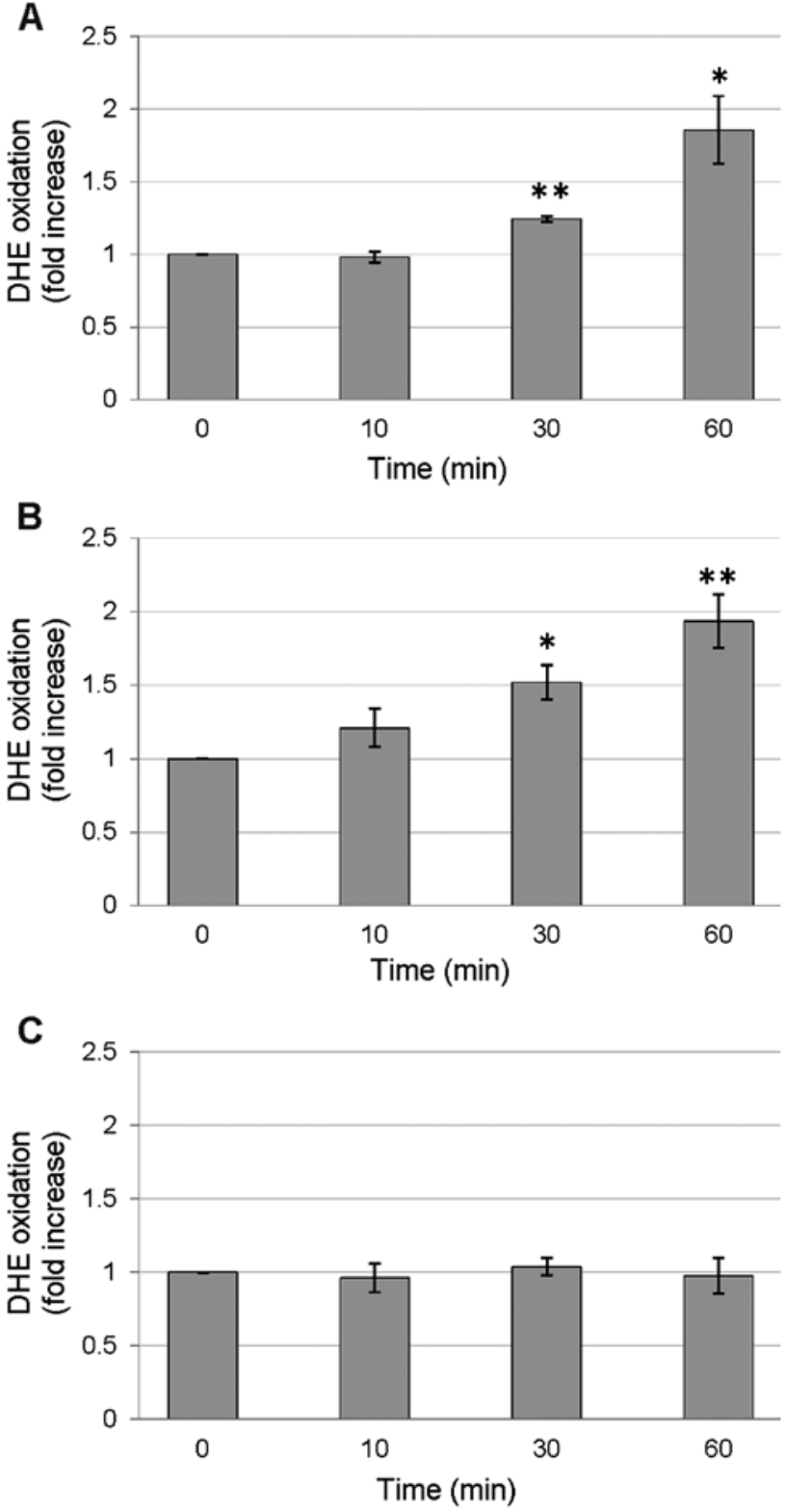

Figure 5. $\mathrm{O}_{2}^{*}$ production in HCT116 cells treated with ATSEE (A), ATTSB (B) or DMSO as a solvent control (C). HCT116 cells were treated with $50 \mu \mathrm{M}$ ATTSB, $50 \mu \mathrm{M}$ ATSEE or DMSO as the solvent control for 0,10 , 30 or $60 \mathrm{~min}$. DHE dissolved in PBS was added to the cells and fluorescence was measured using a Tecan multi-well plate reader. The results represent the average of three independent experiments. ${ }^{*} \mathrm{p}<0.05$ and the result is significant. ${ }^{* *} \mathrm{p}<0.01$ and the result is highly significant.

in intracellular thiol levels is a transient process which is most pronounced at the beginning of the incubation period. Here, the lowest thiol levels were noted after $30 \mathrm{~min}$ for ATTSB and 60 min for ATSEE treatment. Thiol levels then levelled off or even recovered slightly, as in the case of ATTSB after $60 \mathrm{~min}$. It therefore seems that ATTSB and, perhaps to a lesser extent ATSEE, in the first instance interact strongly with cellular thiols, a process which may subsequently result in secondary effects, such as an increase in ROS.

Interestingly, it seems that the loss of thiols actually slightly precedes the increase in ROS concentrations. Indeed, the timeline of events seems to be crucial, and here, an onslaught resulting in a loss of intracellular thiols, most likely by oxida-
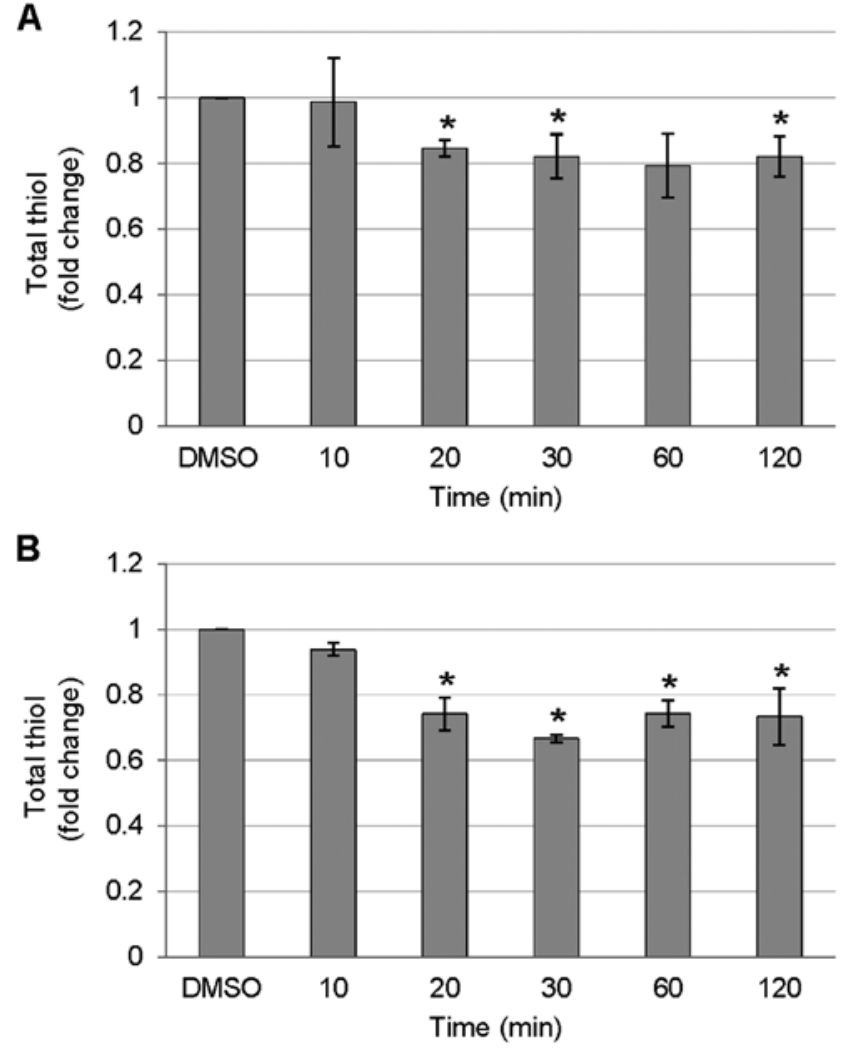

Figure 6. Total thiol concentration of HCT116 cells treated with ATSEE (A) or ATTSB (B). HCT116 cells were treated with $50 \mu \mathrm{M}$ ATSEE or $50 \mu \mathrm{M}$ ATTSB for $0,10,20,30,60$ or 120 min. Cells were collected in PBS, snapfrozen in liquid nitrogen. After thawing and removal of cellular fragments by centrifugation the total thiol concentration was determined by adding Ellman's reagents and measuring the reaction product at $412 \mathrm{~nm}$ in a spectrophotometer.

tion, appears to stand at the beginning. Subsequently, various signalling events may unravel, and here we have focused on some of the most likely suspects which may also explain the 'recovery' of intracellular thiol concentrations observed in this study.

Alterations in the intracellular redox balance often lead to the dissociation of Nrf2 from its inhibitor Keap-1 and a subsequent translocation of $\mathrm{Nrf} 2$ from the cytoplasm into the nucleus (23). In the nucleus, Nrf2 influences a number of processes, including the upregulation of HO-1 expression. The latter provides an adaptive survival response against oxidative stress.

In order to analyse whether ATSEE or ATTSB might influence the level of nuclear Nrf2, we incubated HCT116 cells with ATSEE or ATTSB for different time periods $\leq 24 \mathrm{~h}$. At this point, nuclear extracts were prepared and analysed subsequently on an SDS polyacrylamide gel followed by a western blot analysis with an Nrf2-specific antibody. Interestingly, HCT116 cells treated with ATTSB showed an almost immediate increase of nuclear Nrf2 which remains elevated for $\leq 1 \mathrm{~h}$ after treatment. After that, the level of nuclear Nrf2 returned to the level of the DMSO control (Fig. 7A). Cells treated with ATSEE also showed an increase in the level of nuclear Nrf2, albeit with a delayed onset. It became significant after $1 \mathrm{~h}$ and increased until $2.5 \mathrm{~h}$. Thereafter the level of nuclear Nrf2 returned to a nearly undetectable level (Fig. 7B). Considering 
A
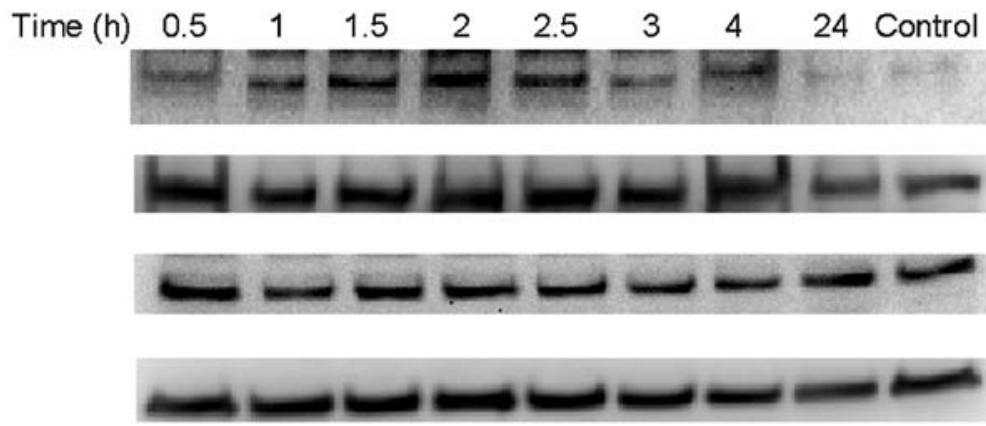

B
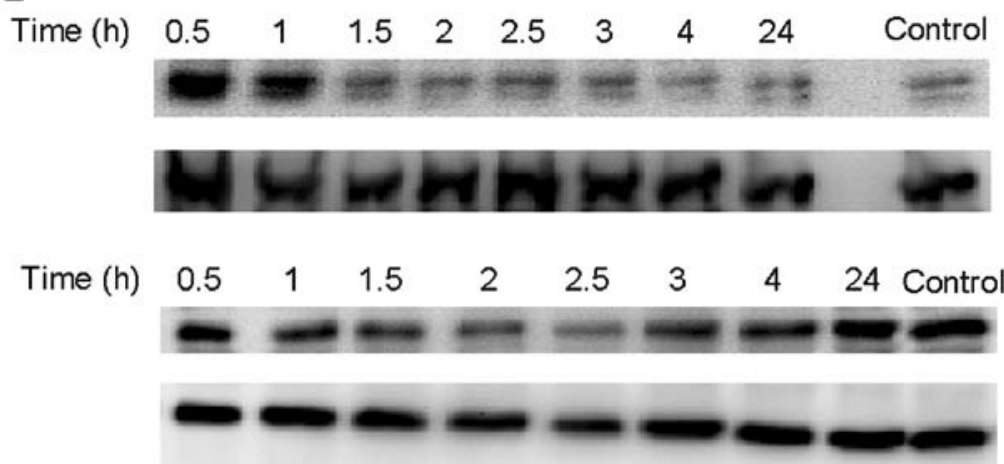

Nrf2 nuclear

nucleolin

Nif2 cytosolic

GAPDH

Nrf2 nuclear

nucleolin

Nrf2 cytosolic

GAPDH

Figure 7. Treatment of HCT116 cells with ATSEE (A) or ATTSB (B) induced nuclear translocation of Nrf2. HCT116 cells were either treated with $50 \mu \mathrm{M}$ ATSEE or ATTSB for $0.5,1,1.5,2,2.5,3,4$ or $24 \mathrm{~h}$. Cytosolic and nuclear proteins were separated on a $10 \%$ SDS polyacrylamide gel and blotted onto a PVDF membrane. Nrf2 was visualised with an appropriate antibody. Nucleolin or GAPDH were used as loading controls. One of at least three independent experiments is shown.

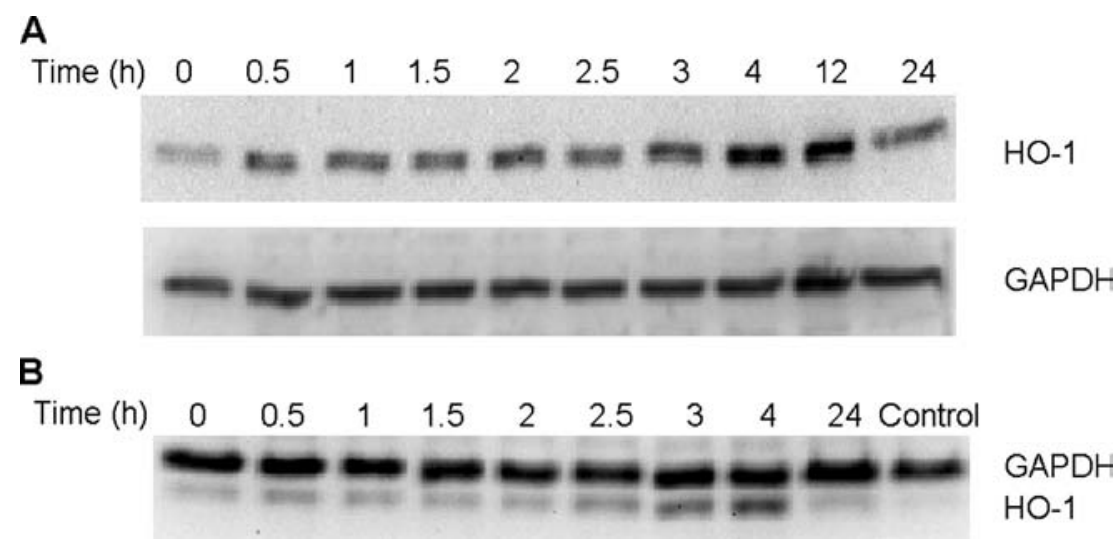

Figure 8. Treatment of HCT116 cells with ATSEE (A) or ATTSB (B) induced an increase in HO-1 protein expression. HCT116 cells were treated with either $50 \mu \mathrm{M}$ ATSEE or $50 \mu \mathrm{M}$ ATTSB for $0.5,1,1.5,2,2.5,3,4,12$ or $24 \mathrm{~h}$. An untreated control $(0 \mathrm{~h})$ was included as baseline situation. Cell extracts were prepared and proteins were separated on a $12.5 \%$ SDS polyacrylamide gel and blotted onto a PVDF membrane. HO-1 was visualised with a HO-1 specific antibody. GAPDH was used as loading control. One representative western blot from three independent experiments is shown.

the different time-dependent changes, it appears that the effects of ATTSB and ATSEE on nuclear Nrf2 mirror the ones on thiol and ROS content. At this point, one may speculate that the two polysulfanes both, although to a different degree, affect the intracellular thiol content, and that such changes trigger an antioxidant response via nuclear translocation of Nrf2 as well as an apparent, more or less simultaneous increase in ROS levels.

One of the downstream targets of Nrf2 in the cellular defence strategy against oxidative stress involves heme oxygenase-1 (HO-1). To analyse whether HO-1 is elevated in HCT116 cells treated with ATSEE or ATTSB, proteins were extracted at specific time points and the extracts were analysed for HO-1 protein expression by western blotting with a HO-1 specific antibody. As shown in Fig. 8A, HCT116 cells treated with ATTSB showed an increase in the level of HO-1 which could be noted after just $0.5 \mathrm{~h}$, with a maximum reached already after 3-4 h and a decline thereafter (Fig. 8B). ATSEE showed a similar, equally rapid increase in the level of HO-1 after just $0.5 \mathrm{~h}$ of treatment. Here, the maximum level of HO-1 
expression was reached after $4 \mathrm{~h}$ and remained elevated until $12 \mathrm{~h}$. It then decreased again.

Changes in HO-1 level observed are in excellent agreement with the ones observed for Nrf2 expression. They further corroborate the hypothesis that compounds such as ATTSB and, to a lesser extent, ATSEE, almost immediately attack the intracellular thiol balance of the cell, an insult which triggers two parallel, and possibly conflicting pathways, one restoring the thiol levels via an antioxidant defence mechanism involving Nrf2 translocation to the nucleus and HO-1 expression, the other causing an increase in intracellular ROS levels, cell cycle arrest and apoptosis. It seems that the nature of the compound employed (e.g., tri- or tetrasulfane) and any possible cellular predisposition to oxidative damage (e.g., cancer cells) ultimately determine which of these processes gains the upper hand.

\section{Discussion}

There is an increasing number of reports which show that natural polysulfanes from garlic exhibit interesting biological activities. The anticancer activity, in particular, seems to be interesting because healthy normal cells are mostly resistant to the treatment with such compounds $(13,16)$. The general activity of these compounds is provided by the polysulfane chain proven by the fact that a replacement of the sulfur chain by a carbon chain results in inactive compounds (18).

In the present study we have employed two derivates consisting of one allyl group (as in the natural product) but two different residues on the other side of the polysulfur chain, namely an ethoxyethyl group in the case of the trisulfane and a benzylgroup in the case of the tetrasulfane. The advantage of these new compounds is that they are nearly odourless which would qualify them for the use in animal cells and in humans. Both new compounds reduced cell viability to a similar extent and comparable to the corresponding natural diallyl polysulfanes. A very interesting aspect is the observation that ATTSB treatment resulted in a complete $\mathrm{G}_{2}$ arrest already after $48 \mathrm{~h}$ whereas the trisulfane ATSEE had no influence on the cell cycle progression. Here, cell cycle analysis already provided some indication for the presence of a sub- $\mathrm{G}_{1}$ population when cells had been treated with ATTSB or with ATSEE, pointing towards possible apoptotic events. By Annexin V staining we could corroborate further that cells treated with either compound go into apoptosis. With respect to the induction of apoptosis, the tetrasulfane ATTSB seemed to be more active than the trisulfane ATSEE.

This led us to the question how such compounds induce apoptosis. Very similar to the natural diallyl polysulfanes, the new asymmetrical compounds also triggered an increase in the intracellular level of $\mathrm{O}_{2}{ }^{-}$radicals and a decrease in the level of total thiols. At the same time, both compounds stimulated the cellular oxidative stress response with an elevation of the amount of $\mathrm{Nrf} 2$ in the nucleus and an elevated expression of heme oxygenase-1 (HO-1). Since HCT116 cells still may enter apoptosis, however, the elevated level of HO-1 alone obviously is not sufficient to ensure that the cells ultimately also survive (and continue to proliferate).

Ultimately, a picture is emerging which shows a fairly swift and decisive cellular response to the polysulfanes employed, whereby the more active tetrasulfane leads to a more rapid and also more pronounced change in the global level of intracellular thiols. Hardly surprising, this assault on the cellular redox balance then activates an antioxidant response, a process which is also fairly rapid and lasting. This antioxidant response involves proteins such as Nrf2 and HO-1 and seems to be able to restore the original thiol level, at least in case of the 'milder' trisulfane ATSEE. In the case of the more aggressive tetrasulfane ATTSB, however, the notable activation of the antioxidant response may not be sufficient to prevent an increase of $\mathrm{O}_{2}^{--}$radicals. The resulting elevated level of oxidative stress and associated damage may dominate the scene, cause a cell cycle arrest and ultimately induce apoptosis. Indeed, these detrimental processes have been observed for ATTSB and also for DATS and DATTS. They are not as pronounced in the case of ATSEE, which may explain why the antioxidant response regains the upper hand and the cells do not arrest or enter apoptosis that much.

In essence, our studies have shown that it is possible to synthesize odourless, asymmetric polysulfanes with comparable ease. These compounds retain the biological activity of their natural counterparts, yet are more amenable to practical applications. Their mode of action is complex, and whilst there does not seem to be one particular cellular target for these sulfur compounds, their action seems to involve two or possibly more parallel pathways. Dominance of one over the other may well result in some selectivity for (cancer) cells, and this is an issue which needs to be explored in more detail as part of future studies. At the same time, ATTSB represents an excellent new lead compound which can be exploited further in the context of various diseases related to a disturbed intracellular redox balance (8). As the chemical synthetic pathways leading to compounds such as ATTSB are comparably straight forward, many derivatives become feasible which will now be designed, synthesized and evaluated as part of the next generation of polysulfanes with medical potential.

\section{References}

1. Block E: Garlic and Other Alliums The Royal Society of Chemistry. Cambridge, 2010.

2. Hofmann AW and Cahours A: Researches on a new class of alcohols. Philos Trans R Soc Lond 147: 555-574, 1857.

3. Semmler FW: Essential oil of garlic (Allium sativum). Arch Pharm 230: 434-448, 1892.

4. Milligan B, Saville B and Swan JM: New syntheses of trisulfides. J Chem Soc 1961: 4850-4853, 1961.

5. Derbesy G and Harpp DN: A simple method to prepare unsymmetrical disulfide, trisulfide and tetrasulfide. Tetrahedron Lett 35: 5381-5384, 1994

6. Czepukojc B, Leroch M, Salm F, Viswanathan UM, Burkholz T, Hahn M and Jacob C: Antifungal activity of tetrasulfanes against Botrytis cinerea. Nat Prod Commun 8: 1599-1603, 2013.

7. Czepukojc B, Baltes AK, Cerella C, Kelkel M, Viswanathan UM, Salm F, Burkholz T, Schneider C, Dicato M, Montenarh M, et al: Synthetic polysulfane derivatives induce cell cycle arrest and apoptotic cell death in human hematopoietic cancer cells. Food Chem Toxicol 64: 249-257, 2014.

8. Marut W, Jamier V, Kavian N, Servettaz A, Winyard PG, Eggleton P, Anwar A, Nicco C, Jacob C, Chéreau C, et al: The natural organosulfur compound dipropyltetrasulfide prevents $\mathrm{HOCl}$-induced systemic sclerosis in the mouse. Arthritis Res Ther 15: R167, 2013.

9. Kelkel M, Cerella C, Mack F, Schneider T, Jacob C, Schumacher M, Dicato M and Diederich M: ROS-independent JNK activation and multisite phosphorylation of Bcl-2 link diallyl tetrasulfide-induced mitotic arrest to apoptosis. Carcinogenesis 33: 2162-2171, 2012. 
10. Jacob C, Battaglia E, Burkholz T, Peng D, Bagrel D and Montenarh M: Control of oxidative posttranslational cysteine modifications: From intricate chemistry to widespread biological and medical applications. Chem Res Toxicol 25: 588-604, 2012.

11. Montenarh M: Diallyl sulfides and the decision about life and death of a cell. In: Natural Compounds as Inducers of Cell Death. Vol. I. Diederich M (ed). Springer Science Business Media, Dordrecht, pp329-344, 2012.

12. Montenarh M and Saidu NEB: The effect of diallyl polysulfanes on cellular signaling cascades. Nat Prod Commun 7: 401-408, 2012.

13. Saidu NEB, Abu Asali I, Czepukojc B, Seitz B, Jacob C and Montenarh M: Comparison between the effects of diallyl tetrasulfide on human retina pigment epithelial cells (ARPE-19) and HCT116 cells. Biochim Biophys Acta 1830: 5267-5276, 2013.

14. Saidu NEB, Valente S, Bana E, Kirsch G, Bagrel D and Montenarh M: Coumarin polysulfides inhibit cell growth and induce apoptosis in HCT116 colon cancer cells. Bioorg Med Chem 20: 1584-1593, 2012.

15. Saidu NEB, Touma R, Asali IA, Jacob C and Montenarh M Diallyl tetrasulfane activates both the eIF $2 \alpha$ and Nrf2/HO-1 pathways. Biochim Biophys Acta 1830: 2214-2225, 2013.

16. Cerella C, Scherer C, Cristofanon S, Henry E, Anwar A, Busch C, Montenarh M, Dicato M, Jacob C and Diederich M: Cell cycle arrest in early mitosis and induction of caspase-dependent apoptosis in U937 cells by diallyltetrasulfide (A12S4). Apoptosis 14: 641-654, 2009
17. Doering M, Ba LA, Lilienthal N, Nicco C, Scherer C, Abbas M, Zada AA, Coriat R, Burkholz T, Wessjohann L, et al: Synthesis and selective anticancer activity of organochalcogen based redox catalysts. J Med Chem 53: 6954-6963, 2010.

18. Busch C, Jacob C, Anwar A, Burkholz T, Aicha Ba L, Cerella C, Diederich M, Brandt W, Wessjohann L and Montenarh M: Diallylpolysulfides induce growth arrest and apoptosis. Int J Oncol 36: 743-749, 2010.

19. Ghibelli L, Fanelli C, Rotilio G, Lafavia E, Coppola S, Colussi C, Civitareale $\mathrm{P}$ and Ciriolo MR: Rescue of cells from apoptosis by inhibition of active GSH extrusion. FASEB J 12: 479-486, 1998.

20. Franco R and Cidlowski JA: SLCO/OATP-like transport of glutathione in FasL-induced apoptosis: Glutathione efflux is coupled to an organic anion exchange and is necessary for the progression of the execution phase of apoptosis. J Biol Chem 281: 29542-29557, 2006.

21. Melino S, Sabelli R and Paci M: Allyl sulfur compounds and cellular detoxification system: Effects and perspectives in cancer therapy. Amino Acids 41: 103-112, 2011.

22. Laragione T, Gianazza E, Tonelli R, Bigini P, Mennini T, Casoni F, Massignan T, Bonetto V and Ghezzi P: Regulation of redox-sensitive exofacial protein thiols in $\mathrm{CHO}$ cells. Biol Chem 387: 1371-1376, 2006.

23. Itoh K, Tong KI and Yamamoto M: Molecular mechanism activating Nrf2-Keap1 pathway in regulation of adaptive response to electrophiles. Free Radic Biol Med 36: 1208-1213, 2004. 